\title{
ESTUDO PSICOLÓGICO LONGITUDINAL NA DISTROFIA MIOTÔNICA
}

\author{
CRISTINA MARIA DUARTE WIGG*, LUIZANTONIO ALVES DURO**
}

\begin{abstract}
RESUMO - Realizou-se estudo longitudinal das alterações cognitivas de 12 casos de distrofia miotônica comparando os resultados de dois exames com intervalo de tempo entre eles, pois em uma primeira avaliação detectaram-se alterações vísuo-espaciais e construtivas que poderiam evoluir ou não com o passar do tempo. Foram aplicados os seguintes instrumentos de medidas psicológicas: matrizes progressivas de Raven (escala especial forma caderno para as crianças, adolescentes e adultos com dificuldades cognitivas graves e escala geral para adolescentes e adultos sem dificuldades cognitivas graves), Wechsler (escala para crianças, subtestes semelhanças e números), cubos de Kohs e Piaget-Head. A seleçāo dos testes e respectivas escalas e formas teve como referencial as entrevistas psicológicas realizadas caso a caso. Estes instrumentos com respectivos resultados foram comparados em cada caso através de duas avaliações, com um intervalo de aproximadamente três anos e meio entre a primeira e a segunda avaliaçōes de cada teste. Quanto ao desempenho nos testes, verificamos: (a) melhor desempenho estatisticamente significante na segunda avaliação pelo teste dos Cubos de Kohs, porém tanto o primeiro quanto o segundo exame denotaram desempenhos bastante insuficientes; (b) leve queda do desempenho no teste de Head 1-3; (c) leve melhora nos demais testes.
\end{abstract}

PALAVRAS-CHAVE: distrofia miotônica, miotonia atrófica, exame psicológico, avaliação longitudinal.

\section{Longitudinal psychologie study in myotonic dystrophy}

SUMMARY - The authors studied in two occasions a group of 12 patients with myotonic dystrophy in a mean interval of three years and a half between the examinations. The neuro - psychological battery included the following tests: Raven's progressive matrices (coloured and general scales), Wechsler children intelligence scale (WISC), Kohs' blocks and Piaget-Head. $50 \%$ of the patients had better scores on the second examination on RCPM, $81.89 \%$ on WISC-digit span, $63.67 \%$ on WISC-numbers, $44.44 \%$ on Piaget-Head 2 and $60 \%$ on Kohs' blocks. However, on Piaget-Head 1-3, the majority had worse results (87.56\%) with statistical significative difference ( $p<0.05$ ). Though the scores from Kohs' blocks were better in $60 \%$ of the patients with $p<0.05$, we have to consider that $60 \%$ had 0 point on first examination being their scores a little better on second one. This is enough to result on statistical significative difference, however, very low if compared to normal subjects.

KEY WORDS: myotonic dystrophy, myotonia atrophica, psychological exam, longitudinal evaluation.

O estudo biomolecular do gene da distrofia miotônica tem apresentado grandes avanços. A partir da descoberta da localização do gene no cromossomo 19 (regiāo q13.3), muitos estudos referentes às ligações gênicas ocorreram. Verkerk et al. ${ }^{34}$ demonstraram que o gene da síndrome do $X$ frágil apresenta diferentes expansões nos diversos doentes. Seguindo este tipo de investigação, Harley et $\mathrm{al}^{16}$ e Buxton et $\mathrm{al}^{4}$ demonstraram que também existiam expansōes do gene na distrofia

Estudo realizado no Setor de Miopatias (SM) do Instituto de Neurologia Deolindo Couto (INDC) da Universidade Federal do Rio de Janeiro (UFRJ) (Diretor: Prof. Dr. Hélcio Alvarenga): * Psicóloga especialista pesquisadora do SM, Professora Auxilar do Departamento de Psicologia da Universidade Gama Filho; ** Professor Adjunto da UFRJ e responsável pelo SM do INDC. Aceite: 15-maio-1995. 
miotônica (DM). A propósito desse assunto, publicamos artigo de atualização referente a tais descobertas $^{12}$. Em nossa avaliaçăo psicológica dos pacientes com D.M., temos verificado com frequência alteraçōes visuo-espaciais e da conduta social. Entre tais acometimentos, observamos diferenças bruscas de humor (depressāo/inibiçāo ou agitaçăo/agressividade) e distúrbios do "saberfazer". As alterações visuo-espaciais e de construção permanecem sendo alvo de nossa atenção no que concerne à investigação dos aspectos cognitivos pois, em publicações anteriores descrevemos diversas alteraçōes psicológicas (distúrbios da atençāo, conceitualizaçāo espacial, abstração e percepção visuo-motora $)^{8,9,36}$. Culebras et al. ${ }^{7}$, a partir das alterações morfológicas que observaram nos cérebros de seus casos, teceram comentários quanto ao acometimento talâmico que poderia estar relacionado à grande dificuldade dos pacientes para construir formas a partir de modelos.

Como em estudos anteriores que objetivaram analisar a possibilidade de diminuição do quociente intelectual através dos anos ${ }^{32,39}$ não se chegou a conclusões definitivas, resolvemos realizar o estudo longitudinal para verificar se há degeneração progressiva, o que nos permitiria ressaltar a presença de deterioração progressiva das funçōes mentais através da evoluçāo do quadro clínico.

\section{METODOLOGIA}

Separou-se ao acaso um grupo de 12 pacientes com DM. Os casos variaram de acordo com a faixa etária ( 7 a 50 anos, com média de $26,67 \pm 11,81$ ), sexo ( 6 masculinos e 6 femininos), tempo de doença (de 7 a 45 anos, média de $20,17 \pm 12,83$ ) e nível de instrução. $O$ intervalo médio entre a primeira e a segunda avaliação foi 3,08 $\pm 1,44$ anos. Dos 12 pacientes, 12 realizaram a primeira e segunda avaliações com o teste de Raven ${ }^{26,27}$ (- escala especial e geral) 11 o WISC semelhanças e números ${ }^{21,35}, 10$ os cubos de Kohs $^{20}$ e 9 o Piaget-Head ${ }^{38}$

O diagnóstico da DM foi feito a partir da anamnese e do exame clínico-neurológico do paciente, avaliação do tipo de herança familiar, estudo eletroneuromiográfico, exames oftalmológico, cardiovascular, psicológico e fonoaudiológico, testes de funçāo pulmonar e enzimática. Em alguns casos foram realizados também estudos endócrinos e tomográficos.

Foram comparados os resultados da primeira e segunda avaliações dos seguintes testes: matrizes progressivas de Raven (escala especial e geral conforme o caso), escala de inteligência Wechsler ${ }^{35}$ para crianças (subtestes semelhanças e números do WISC), cubos de Kohs e Piaget-Head.

Os dados utilizados na Tabela 1 referiram-se aos resultados brutos nos testes de Raven (especial e geral), cubos de Kohs , Piaget-Head e WISC. Os resultados brutos foram utilizados no Raven (especial e geral) e cubos de Kohs com o objetivo de não convertermos os resultados originais segundo tabelas não-adaptadas ou nãorevisadas, o que poderia descaracterizar nossa amostra. Os resultados do teste de Piaget-Head sāo analisados comumente em sua forma bruta. Quanto ao WISC, foi utilizada a escala adaptada pela PUC-Rio de Janeiro ${ }^{21}$. porém as notas brutas não foram convertidas em ponderadas para que o procedimento fosse uniforme.

A fim de verificar se havia diferença significativa entre os resultados das duas avaliações utilizou-se o teste de Wilcoxon para amostras relacionadas. Para cada instrumento de medida foram relacionados os dois resultados (primeira e segunda avaliações), com o objetivo de detectar diferenças significativas. A diferença só foi considerada significativa com grau de confiança de $95 \%$ ou maior ( $p$ igual ou menor que 0,05 ).

\section{RESULTADOS E COMENTÁRIOS}

Alterações morfológicas do sistema nervoso na DM tem sido demonstradas desde o trabalho de Steinert, em 1909, que fez a análise anátomo-patológica de um de seus $\operatorname{casos}^{30}$. Despovoamento neuronal, aumento das cavidades ventriculares e inclusões eosinofílicas ocorreram em um dos nossos $\operatorname{casos}^{11}$, alterações estas também descritas por outros autores ${ }^{7,24.28 .37}$. Na avaliação através de ressonância magnética e tomografia computadorizada, Glantz et al. ${ }^{13}$ encontraram aumento nas cavidades ventriculares e hiperdensidade periventricular.

Estudos eletrofisiológicos utilizando técnicas de potenciais evocados (auditivos de tronco cerebral e de média latência, visual, eletrorretinografia e sômato-sensorial $)^{6.14,23,25,29,33}$, demonstraram alterações nos exames oculomotor e vestibular. A utilização da técnica de obtenção da onda P300 
Tubela 1. Relação dos casos e respectivas pontuações (resultados brutos) da primeira e da segunda avaliação.

\begin{tabular}{|c|c|c|c|c|c|c|c|c|c|c|c|c|}
\hline \multirow{2}{*}{$\begin{array}{c}\mathrm{Ca} \\
1\end{array}$} & \multicolumn{2}{|c|}{ MPR } & \multicolumn{2}{|c|}{$\begin{array}{c}\text { WISC } \\
\text { se }\end{array}$} & \multicolumn{2}{|c|}{$\begin{array}{c}\text { WISC } \\
\text { nu }\end{array}$} & \multicolumn{2}{|c|}{ KOHS } & \multicolumn{2}{|c|}{$\mathrm{PH}-2$} & \multicolumn{2}{|c|}{ H -1.3} \\
\hline & 11 & 12 & 3 & 6 & 7 & 6 & 0 & 0 & 22,0 & 13 & 14.5 & 14.0 \\
\hline 2 & 14 & 12 & 13 & 15 & 8 & 8 & 3 & 3 & 31,0 & 32 & 34.0 & 28.0 \\
\hline 3 & 7 & 13 & 11 & 7 & 7 & 8 & 0 & 3 & 20,0 & 16 & 23.5 & 13.5 \\
\hline 4 & 19 & 14 & 18 & 19 & 7 & 10 & 2 & 0 & 34,0 & 34 & 40.0 & 40.0 \\
\hline 5 & 57 & 54 & 18 & 21 & 11 & 15 & 131 & 133 & 34,0 & 35 & 3.0 & 46.0 \\
\hline 6 & 49 & 47 & 7 & 10 & 10 & 11 & 70 & 79 & 34,0 & 34 & 30.5 & 29.5 \\
\hline 7 & 22 & 23 & 10 & 9 & 10 & 11 & 21 & 2 & 28,0 & 35 & 28.0 & 23.0 \\
\hline 8 & 19 & 19 & 12 & 16 & 11 & 13 & 3 & 8 & 31,0 & 31 & 38.0 & 28.5 \\
\hline 9 & 30 & 44 & 5 & 9 & 8 & 9 & 3 & 28 & 11,5 & 21 & 18.5 & 10.5 \\
\hline 10 & 17 & 13 & 5 & 8 & 6 & 0 & 28 & 28 & & & & \\
\hline 11 & 14 & 14 & 9 & 10 & 12 & 11 & & & & & & \\
\hline 12 & 27 & 28 & & & & & & & & & & \\
\hline Med & 24 & 24 & 11 & 12 & 9 & 9 & 26 & 36 & 27 & 28 & 30 & 26 \\
\hline DP & 15 & 15 & 5 & 5 & 2 & 4 & 43 & 43 & 8 & 9 & 10 & 12 \\
\hline To & 286 & 293 & 111 & 130 & 97 & 102 & 261 & 305 & 245 & 251 & 270 & 233 \\
\hline
\end{tabular}

Ca casos; Med, média; DP, desvio padräo; To, somatório dos pontos dos grupos; MPR, matrizes trizes progressivas de Raven; se, semelhanças; nu, números; PH, Piaget-Head; KOHS, cubos de Kohs.

$O$ resultado da primeira coluna corresponde ao primeiro teste e o da segunda ao teste realizado com um determinado intervalo de tempo

(gerada quando o paciente discrimina diferentes estímulos auditivos) ${ }^{15}$ levou à conclusão de que havia lenificação em 6 dentre $8 \mathrm{DM}$. Isto confirmaria a presença de alterações cognitivas e no processamento da informação decorrente de anormalidades no desenvolvimento cerebral.

Estudos psicológicos têm sido desenvolvidos em diversas formas de miopatias. Bryan ${ }^{3}$ comparou 13 adolescentes com distrofia muscular forma fascio-escápulo-umeral com 11 normais, utilizando-se do Wechsler. Concluiu que o grupo com distrofia tinha quociente intelectual (QI) inferior, com muitas variações individuais. Karagan e Sorensen ${ }^{18}$ avaliaram diversos casos de distrofia muscular de Becker, fascio-escápulo-umeral e cinturas (utilizaram a escala de inteligência para crianças do teste de Wechsler - WISC). Concluíram que o QI verbal era inferior ao de desempenho (não verbal). Os pacientes mais graves eram os que apresentavam piores resultados, independentemente do tipo de distrofia.

$\mathrm{Na} \mathrm{DM}$ temos verificado deficiência na capacidade cognitiva, que nāo necessariamente piora com o tempo. Tal déficit independe da faixa etária ou do sexo e, provavelmente, está relacionado ao tipo ou extensāo da disfunçāo cerebral, além das influências sofridas pelas limitaçōes psicossociais. Sendo assim, devemos nos ater aos dados que, com casuísticas e abordagens diferentes, comprovam que a cognição está atingida, confirmando os achados mais remotos dos estudos psicológicos de Branwell Addis $^{2}$, Maas e Paterson ${ }^{22}$, Thomasen ${ }^{31}$, Klein ${ }^{19}$, Rosman e Kakulas ${ }^{28}$, Harper ${ }^{17}$ e Wsniewski et $\mathrm{a}^{37}$, dentre outros. 
Tabela 2. Resultados do teste de Wilcoxon frente aos dados brutos da primeira e da segunda avaliação dos testes psicológicos.

\begin{tabular}{lccccc}
\hline Teste psicológico utilizado & Total & Média meses & DP & \multicolumn{2}{c}{ Wilcoxon } \\
& & & & Valor de Z & Valor de P \\
\hline \hline RAVEN & 12 & 36.41 & 21.70 & .31 & .75 \\
WISC semelhanças & 11 & 39.45 & 21.36 & 1.87 & 06 \\
WISC números & 11 & 39.45 & 21.36 & 1.15 & 24 \\
CUBOS DE KOHS & 10 & 41.40 & 21.48 & 2.29 & .02 \\
PIAGET-HEAD 2 & 9 & 41.77 & 22.75 & 1.01 & .31 \\
HEAD 1-3 & 9 & 41.77 & 22.75 & 2.19 & .03 \\
\hline
\end{tabular}

Total, total de pacientes; Média, tempo médio entre os dois exames, em meses; $D P$, desvio padrão da média

Conforme os resultados da Tabela 2, nas matrizes progressivas de Raven não houve diferença significativa $(p=0,75)$. Ao analisarmos os resultados brutos (Tabela 1), verificamos que o desempenho melhorou em $50 \%$ dos casos, ao contrário de nossas expectativas.

No WISC-semelhanças, os escores da segunda avaliação, quando comparados aos da primeira, estavam aumentados em $81,89 \%$ dos casos. Em $18,11 \%$, não houve alteração do escore. Não foi verificado qualquer caso de rebaixamento do escore e nem houve diferença significativa $(p=0,06)$.

Os resultados do WISC-números revelaram que na segunda avaliação os escores estavam aumentados em $63,67 \%$, inalterados em $9,99 \%$ e diminuídos em $18,11 \%$. Concluiu-se que neste instrumento o teste de significância não revelou diferença significativa $(p=0,25)$.

No Piaget-Head 1-3 observou-se deterioração em $87,5 \%$ dos casos, resultados inalterados em $11,11 \%$, não havendo caso de aumento de escore na segunda avaliaçāo. A lateralidade por aspectos visuo-espaciais denotou deterioração ao longo dos anos. Ocorreu diferença significativa $(p=0,03)$.

Nos resultados do Piaget-Head 2, apenas dois casos $(22,22 \%)$ apresentaram seus escores rebaixados. Quanto aos demais, 44,44\% mantiveram seus resultados e 33,33\%, melhoraram. Aspectos conceituais da lateralidade não se deterioraram, não havendo perda dos conhecimentos de lateralidade (direita-esquerda). Não se detectou diferença significativa $(p=0,31)$.

Os resultados dos testes de Raven, WISC e Piaget-Head 2 não demonstraram queda significativa do desempenho ao longo dos anos, o que contraria as afirmações de Maas e Paterson ${ }^{22}$ e confirma as de Bird et al.' 'quanto à deterioração progressiva destas características.

Podemos verificar que há possibilidade de melhora das funçōes cognitivas, embora o ganho seja mínimo diante da limitação apresentada e não se possa afirmar que seja significativo.

Intervir no processo de desenvolvimento cognitivo de miopatas, poderia se transformar numa alternativa de estimulação e melhora deste processo.

Nos cubos de Kohs (considerado por nós como o mais importante exame psicológico em DM, pois temos demonstrado, ao longo das avaliações desses casos, que esta é a mais notável dificuldade do grupo de pacientes) foi verificada diferença significativa $(p=0,02)$, às custas de melhora do desempenho no segundo teste.

Devemos enfatizar que $60 \%$ dos casos não conseguiram sequer resolver satisfatoriamente o primeiro item do teste, demonstrando dificuldades de compreensāo, análise e síntese, além de séria dificuldade do "saber-fazer". Dos casos que realizaram de alguma forma o teste, $60 \%$ melhoraram o 
desempenho e $10 \%$, pioraram. Em $20 \%$ os resultados não se alteraram. Sendo assim, consideramos que nāo houve neste intervalo de tempo deterioração em nível de conceitualização espacial e das funções vísuo-espaciais. Porém, como já referido anteriormente, o desempenho nesse teste já era bastante insatisfatório na primeira avaliação.

Os resultados do Piaget-Head 2 e dos cubos de Kohs, independentemente da avaliação longitudinal, denotam a precariedade das funções vísuo-espaciais e vísuo-motoras. Segundo os estudos de Penez (1978) e Walker et al. (1984), alterações vísuo-espaciais e vísuo-motoras são frequentes nos casos de DM (apud Censori et al. ${ }^{5}$ ) Censori et al. ${ }^{5}$ também consideraram as alteraçōes vísuo-espaciais e de construção como importantes no estudo cognitivo da DM, utilizando o teste dos cubos de Kohs.

\section{CONCLUSÃO}

Conforme podemos perceber, quatro de nossas avaliações (MPR, WISC semelhanças e números e Piaget-Head 2) denotaram melhora da capacidade. O resultado do teste Piaget-Head 1-3 caracterizou deterioração progressiva da lateralidade por aspectos vísuo-espaciais. Este resultado está de acordo com o péssimo desempenho nos cubos de Kohs, também por aspectos vísuo-espaciais, apesar de nāo termos verificado deterioração progressiva.

Consideramos que nossa amostra deve ser aumentada para que possamos obter conclusão mais definitiva quanto aos resultados. Também objetivamos aumentar o período entre a primeira e a segunda avaliações, a fim de estudar a diferença através de período mais longo.

\section{REFERÊNCIAS}

1. Bird TD; Follett C, Griep E. Cognitive and personality function in myotonic muscular dystrophy. J Neurol Neurosurg Psychiatry 1983, 46:971-980.

2. Bramwell E, Addis WR. Myotonia atrophica. Edinburgh Med J 1913,11: 21-44.

3. Bryan GE. Psychological characteristics of adolescents in a kindred known to have fascio-scapulo-humeral muscular dystrophy (Doctoral dissertation, University of Utah, 1958) (University Microfilms $\mathbf{N}^{0} .58-7608$ ). Diss Abs Int 1959, 19:2653-2654.

4. Buxton J, Shelbourne P, Davies J. Detection on an unstable fragment of DNA specific to individuals with myotonic dystrophy. Nature 1992, 355:547-548.

5. Censori B, Danni MP, Provinciali L. Neuropsychological profile in myotonic dystrophy. J Neurol 1990 , 237:251-256.

6. Cosi V, Bergamaschi R, Versino M. Callieco R; Sandrini G, Ruiz L. Multimodal evoked potentials in myotonic dystrophy. Neurophysiol Clin. 1992, 22:41-50.

7. Culebras A, Feldman RG, Merck FB. Cytoplasmic inclusion bodies within neurons of the thalamus in myotonic dystrophy: a light and electron microscope study. J Neurol Sci 1973, 19:319-329.

8. Duarte CMO. Aspectos psicológicos da distrofia miotônica. IX Jornada de Iniciação Científica. UFRJ. Rio de Janeiro, outubro 1986, p 200

9. Duarte CMO, Hora LHM, Duro LAA. Estudos psicológicos na doença de Steinert. V Jornada de Miopatias. Rio de Janeiro, outubro 1985, p 2.

10. Duarte, CMO, Hora LHM, Duro LAA. Avaliação psicológica na Doença de Steinert. Rev Bras Neurol 1986, 22:39-42

11. Duro LAA. Distrofia miotônica. Tese, UFRJ. Rio de Janeiro, 1985.

12. Duro LAA, Penque GMCA. Distrofia miotônica. Rev Bras Neurol 1993, 29:69-70.

13. Glantz RH, Wright RB, Huckman MS, Garron DC, Siegel IM. Central nervous system magnetic resonance imaging findings in myotonic dystrophy. Arch Neurol 1988, 45:36-37.

14. Gott PS, Larmaze DS, Keane JR. Abnormal visual evoked potentials in myotonic dystrophy. Neurology $1983,33: 1622-1625$.

15. Hanafusa H, Motomura N, Asaba H, Sakai T, Kawamura H. Event-related potentials (P 300) in myotonic dystrophy. Acta Neurol. Scand., 1989, 80:111-113.

16 Harley HG, Brook JD, Rundle SA. Expansion of an unstable DNA region and phenotypic variation in myotonic dystrophy. Nature 1992, 355:545-546.

17. Hasper PS. Congenital myotonic dystrophy in Britain: I. Clinical aspects. Arch Dis Child 1975, 50:503-513.

18. Karagan NJ, Sorensen JP. Intellectual functioning in non-Duchenne muscular dystrophy. Neurology, 1981, $31: 448-452$. 
19. Klein D. La dystrophie myotonique (Steinert) et la myotonie congenitale. (Thomsen) en Suisse. J Gen Hum 1958, 1 (Suppl): 1-328.

20. Kohs SC. Manual do teste dos cubos de Kohs. Rio: Centro Editor de Psicologia Aplicada (CEPA), 1970.

21. Lemgruber V, Paine PA. Adaptação brasileira da escala verbal do WISC. Arq Bras Psicol, 1981, 33:32-56.

22. Maas O, Paterson AS. Mental changes in families affected by dystrophia myotonica. Lancet 1973, 1:21-23.

23. Mendes MFX. Potenciais cerebrais evocados na distrofia miotônica. Tese, UFRJ Rio de Janeiro, 1992.

24. Ono S, Inoue K, Mannen T, Kanda F, Jinnai K, Takahashi K. Neuropathological changes of the brain in myotonic dystrophy: some new observations.J Neurol Sci 1987, 81:301-320.

25. Pinto F, Amantini A, Scisciolo G, Scaioli V, Frosini R, Pizzi A, Marconi G. Electrophysiological studies of the visual system in myotonic dystrophy. Acta Neurol Scand 1987, 76:351-358.

26. Raven JC. Teste das matrizes progressivas: escala geral. Rio: CEPA, 1977.

27. Raven JC. Test de matrices progresivas para la medida de la capacidad intelectual: escala especial. Buenos Aires: Paidos, 1986.

28. Rosman NP, Kakulas BA. Mental deficiency associated with muscular dystrophy: a neuropathological study. Brain 1966, 89:769-786.

29. Sandrini G, Gelmi C, Rossi V, Bianchi PE, Alfonsi E, Pachetti C, Verri AP, Nappi G. Electroretinographic and visual evoked potentials abnomalities in myotonic dystrophy. Electroenceph Clin Neurophysiol 1986, 64:215- 217.

30. Steinert $\mathbf{H}$. Uber das klinische und anatomische Bild des Muskelschwunds der myotoniker. Deutsch $\mathrm{Z}$ Nervenheilk 1909, 37:58-104.

31. Thomasen E. Myotonia. Denmark : Universitsforlaget Aarhus, 1948.

32. Vanier TM. Dystrophia myotonica in childhood. Br Med J 1960, 2:1284-1288.

33. Verhagen WIM, ter Bruggen JP, Huygen PLM. Oculomotor, auditory, and vestibular responses in myotonic dystrophy. Arch Neurol 1992,49:954-960.

34. Verkerk A, Pierletti M, Sutcliffe J. Identification of a gene (FMR-1) containing a CGG repeat coincident with a breakpoint cluster region exhibiting length variation in fragile X syndrome.Cell 1991, 65:905-914.

35. Wechsler D. Escala da inteligência Wechsler para crianças (WISC). Tradução de Ana Maria Poppovic. In: Manual de Psicologia Aplicada São Paulo: Centro Editor de Psicologia Aplicada, 1977.

36. Wigg CMD, Duro LAA. Avaliação psicológica em pacientes com distrofia miotônica. XIV Congresso Brasileiro de Neurologia, Rio de Janeiro, setembro 1990. In Arq Neuro-Psiquiatr, 1990, 48 (Supl): 388.

37. Wisniewski HM, Berry K, Spiro AJ. Ultrastructure of thalamic neuronal inclusions in myotonic dystrophy. J Neurol Sci 1975, 24:321-329.

38. Zazzo R. Manual para o exame psicológico da criança. Ed 2. Sāo Paulo: Mestre Jou, 1981, Vol 1.

39. Zellweger H, Jonasescu V. Early onset of myotonic dystrophy in infants. An J Dis Child 1973, 125: 601-604. 